\title{
Cooperative Control of Large Systems
}

\author{
Maziar E. Khatir ${ }^{1} \quad$ Edward J. Davison ${ }^{2}$ \\ ${ }^{1}$ Systems Control Group Dept. of Electrical and Computer Engineering, Univer- \\ sity of Toronto, Toronto, Ontario M5S 3G4 Canada, mkhatir@control.utoronto.ca \\ ${ }^{2}$ Systems Control Group Dept. of Electrical and Computer Engineering, Univer- \\ sity of Toronto, Toronto, Ontario M5S 3G4 Canada, ted@control.utoronto.ca
}

\section{Abstract}

"Cooperative control" is a term which is used to capture those problem areas in which some type of repetition of identical or non-identical subsystems, which are interconnected together, occurs. Such systems are often found in nature, i.e. in the motion of clusters of birds, fish, insects, etc. moving together, in the cell structure of mammals and life-forms, and also in the man-made systems such as in transportation systems. In such systems, a decentralized control configuration is often applied to control the overall system, so that some common objective is achieved.

It is perhaps too early to be able to list the fundamental problems arising in this problem area; there is however one important application area in which an understanding of the behaviour of the near-identical objects is desirable, and that is with respect to disease processes associated with the abnormal behaviour of certain organs of the human body.

This paper gives a review of such typical example systems for the case of:

- "fish-like" objects interacting in water.

- vehicles which have the front wheel drive / steering interacting in a string formation.

- analyzing the behaviour of intestinal slow-wave patterns which occur in the human body.

\section{Introduction}

In large systems which are made of identical or near-identical sub-systems called "agents", cooperation plays a critical role; in such systems, as is often the case in control system design, stability, transient time response, and robustness are the most important terms to be considered. There are however additional properties which need to be considered, and these will be discussed later.

The agents of a large system often interact with each other, via an interconnection topology which exists between these agents. In most cases, the agents are 
autonomous and self driven, and so it can be expected that their interconnection topology may not be fixed, which makes the analysis and controller design of such systems more complicated. However in some classes of large systems, the interconnection structure is fixed and it can be assumed that it will not change under normal conditions. Examples of systems with time-varying interconnection structure are schools of fish, groups of bees, and flocks of birds, etc. (It is to be noted that birds can also fly in a fixed formation.) Examples of systems with a fixed interconnection structure consist of a platoon of vehicles in a highway, the intestinal system in the human body, flight formation, etc. For interconnected systems, in addition to the usual stability and robustness analysis, other properties become important and must be considered, such as "string stability" [1], [2], "connectivity of interconnection network", and "convergence of the clusters". These terms exist only for so-called multi-agent systems which contain identical or near-identical subsystems, and are not defined for conventional control systems. Large systems consisting of near-identical objects can often be found in nature. For example, in the 1960's the intestine of a mammal was considered as a fixed interconnection structure multi-agent system consisting of a cascade of oscillators [3], [4]. In the early 1970's, the modeling and simulation of multi-neuron networks was considered as a cooperative multi-agent system with respect to the modeling of respiratory rhythm and the lateral inhibition of the eye [5]. On the other hand, many contributions have been made by biologists in studying the behaviour of groups of animals, insects, birds, fish etc as a variable interconnection structure system. More recently, there has been close cooperation between engineers and biologists which has opened windows for better modeling and understanding of the complicated and weird behaviours of creatures in nature [6]; stability analysis and connectivity and convergence of clusters have been also considered by many researchers [7], [8], [9]. In addition to natural systems, many man-made multi-agent systems which have been developed in the $20^{\text {th }}$ century have a fixed interconnection topology, e.g. transportation systems.

In this paper, three examples of different types of multi-agent systems are studied and simulated to illustrate the different types of behaviour which may occur in this exciting area of research.

\section{Cooperative Control Systems}

"Cooperative Control" is a term which is used to capture those problem areas in which some type of repetition of identical or non-identical subsystems, which are interconnected together, occurs. Such systems can be found:

- In Nature: e.g. Birds, Fish, Insects moving together

- In Man-made Systems: e.g. Transportation problems, Network systems

- In various organs of the human body: e.g. Intestinal system 2.1 .

In this case, various types of interconnection patterns may arise, i.e. see figure 


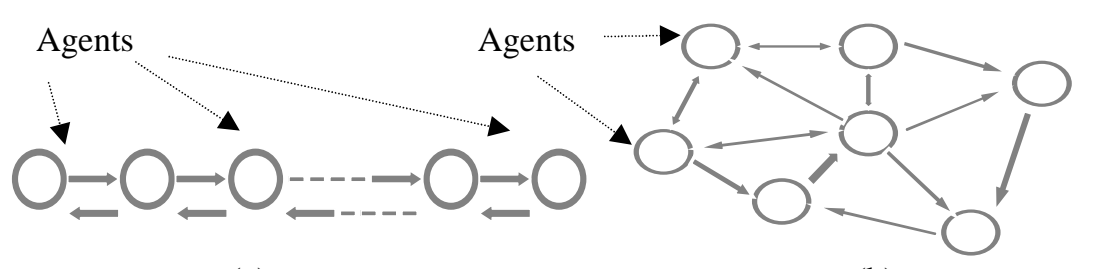

(a)

(b)

Fig 2.1. Agents may interact in a cascade structure (a) or in a neighbourhood structure (b).

It is desired to develop an understanding of the behaviour of such interconnected systems; in particular:

- when the number of subsystems $N$ is very large.

- when the control of such interconnected system is constrained to be decentralized.

This paper gives a brief overview of some preliminary studies for three cases of cooperative control systems which occur:

- In nature

- In man made systems

- In organs of the human body

\section{Example of Cooperative Control Occurring in Nature}

In this example, we study the behaviour of a group of "fish" which are swimming in water, under different control configurations.

\subsection{Modeling of a "fish"}

Each fish in the group is assumed to have the same model and is modeled by a nonlinear dynamical system with a non-holonomic constraint. There are three dynamical states $v, \omega, \psi$ and three kinematical states $x, y$, and $\theta$ for this model as given in figure 3.1; the state space dynamical equations for this model are given by:

$$
\left\{\begin{array}{l}
\dot{\psi}=-\omega+\frac{1}{m v} F \sin \psi \\
\dot{v}=-\frac{B(\psi)}{m} v+\frac{1}{m} F \cos \psi \\
\dot{\omega}=-\frac{b}{J} \omega+\frac{1}{J} \tau \\
\dot{x}=v \cos (\theta+\psi) \\
\dot{y}=v \sin (\theta+\psi) \\
\dot{\theta}=\omega
\end{array}\right.
$$


where $v, \omega, \psi, m, J, b$ are respectively the velocity, angular velocity, skidding angle, mass, moment of inertia, and coefficient of rotary damping of the fish, and $F$ and $\tau$ are independent control input signals where $F$ is the traction force and $\tau$ is the rotary torque input. $B(\psi)$ is the viscous friction coefficient which depends highly on the shape of the object; in this case, we will assume that the viscous friction coefficient is proportional to the surface exposed to the medium, so that it can be approximated by:

$B(\psi)=B_{1}|\cos \psi|+B_{2}|\sin \psi| \cdot$

for the case of the traction force and rotary torque signals given in figure 3.3 (a).

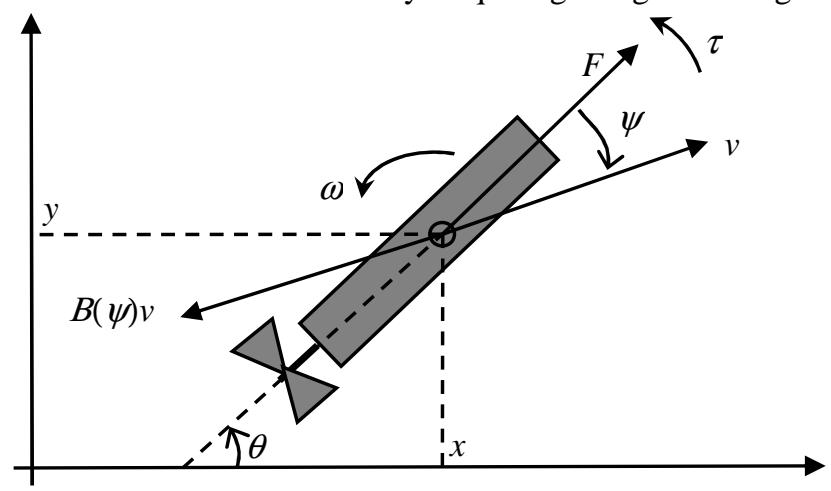

Fig 3.1. Model of the underwater object

\subsection{Control of "fish"}

Here it is assumed that each "fish" has a local controller consisting of two parts:

- a Cascade Controller

- a Neighbourhood Controller

A description of these two controllers is given as follows.

\subsubsection{Cascade Control}

Here we assume that the "fish" are constrained to exist in a two dimensional plane, and that each "fish" has a local controller which tracks a reference signal for the velocity and heading angle. This controller must be able to track constant velocity, and constant heading angle references without steady state error. The velocity of each agent is controlled by the traction force $F$, and the heading angle is controlled by the rotary torque $\tau$ using two separate controllers (see figure 3.2). In this case, each "fish" follows the velocity and heading angle references provided by the neighbourhood controller (described in section 3.2.2). 


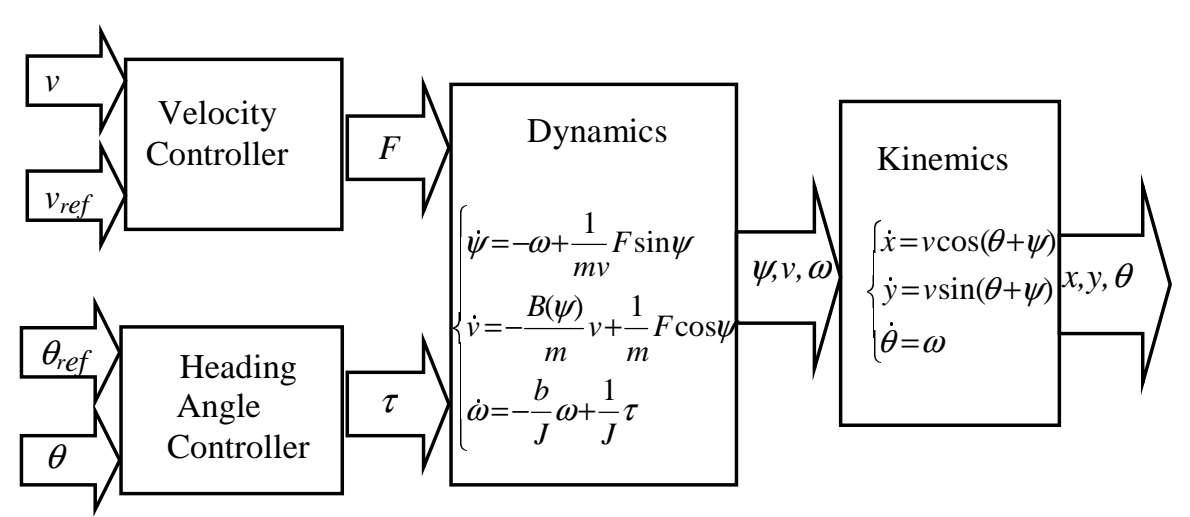

Fig 3.2. The local tracking controller for each individual agent

The response of the closed loop system due to step function changes in the velocity reference and heading angle reference for a single "fish" is given in figure 3.3, plot (b).
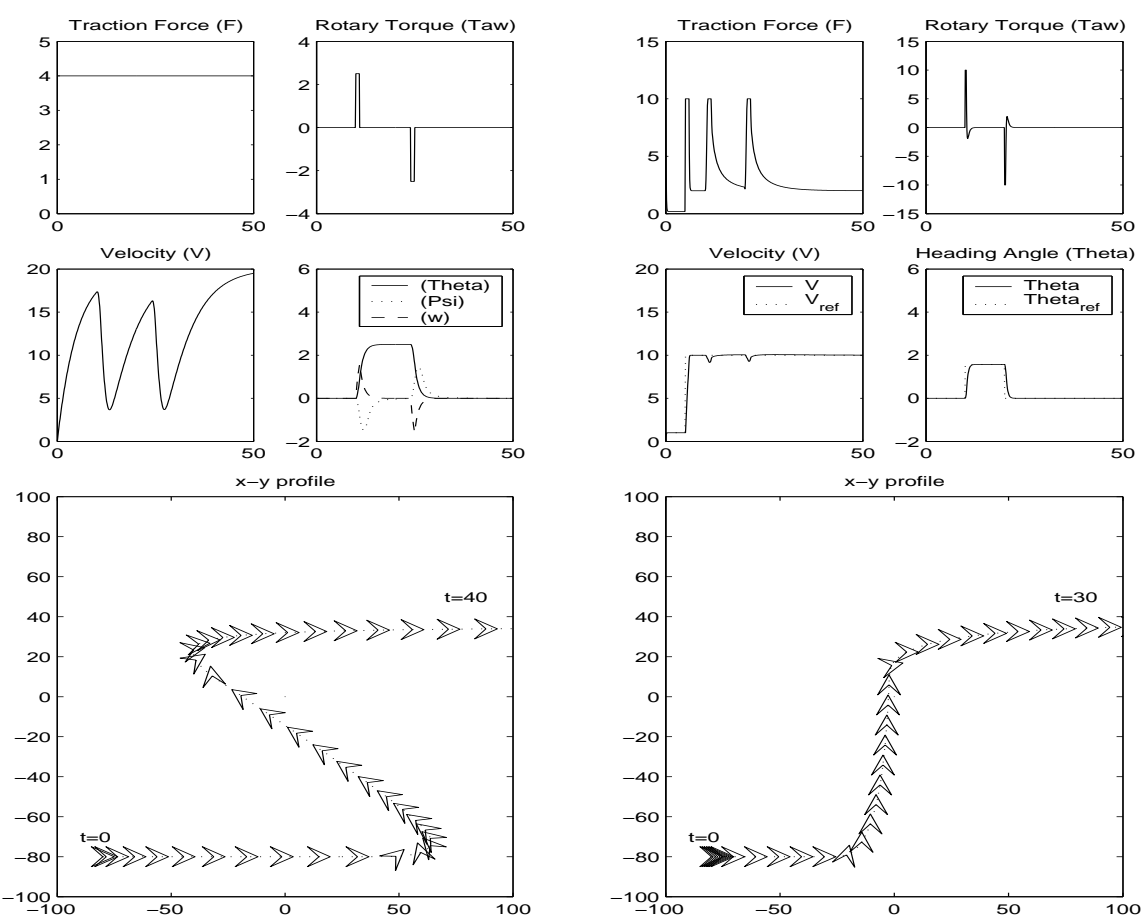

(a)

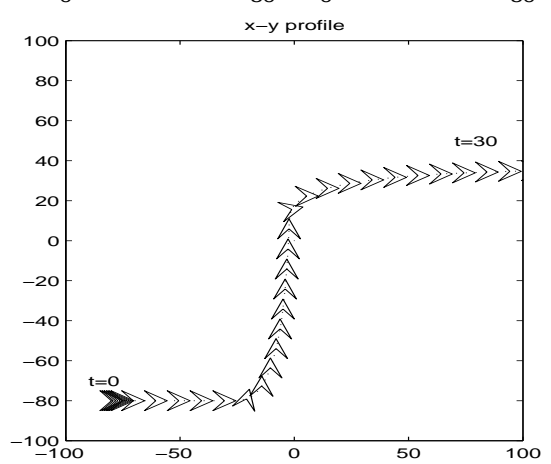

(b)

Fig 3.3. Response of a single "fish" to a constant traction force and a rotary torque input (feed forward control) (a), and the response of the same "fish" under closed loop control for a step function reference input for the velocity (which occurs at $t=5$ ), and for the heading angle (which occurs at $t=10$ and $t=20$ ) (b). 


\subsubsection{Neighbourhood Control}

In this section, we consider a collection of $\mathrm{N}$ "fish" in a two dimensional plane which have an arbitrary position and orientation. Here it is assumed that there is a single "lead fish" which, independently of the other fish, selects its own velocity and direction trajectory, and it is desired to obtain a decentralized controller for the fish in the group, so that these fish will follow the trajectory of the lead fish. In this case, the cascade controller as discussed in the previous section is applied to each "fish" of the group, with a neighbourhood controller providing the reference velocity $v^{r e f}$, and reference direction $\theta_{i}^{\text {ref }}, i=1,2, \ldots, N$, which is determined by the average velocity and direction of "fish" in a neighbourhood of the fish. In particular, the neighbourhood controller relies on two types of behaviour in order to specify its desired tracking direction and desired velocity, averaging, and attraction / repulsion, which is described as follows.

\section{Averaging}

Averaging is a simple and effective method to find the appropriate velocity and heading angle for each agent. Here it is assumed that, each agent has a vision range or a so-called "radius of neighborhood" and adjusts its reference direction and reference velocity by averaging the settings of all agents inside this neighborhood region including itself. These settings can be either the heading angle or the velocity or both. Mathematically:

$$
\begin{array}{cc}
v_{i}^{\text {ref }}(t+1)=\frac{v_{i}(t)+\sum_{j \in\{\text { Neighbors of Agent } \mathrm{i}\}} v_{j}(t)}{1+\{\text { Number of neighbours of Agent i }\}} & i=1,2, \ldots, N \\
\theta_{i}^{\text {ref }}(t+1)=\frac{\theta_{i}(t)+\sum_{j \in\{\text { Neighbors of Agent i }\}} \theta_{j}(t)}{1+\{\text { Number of neighbors of Agent } \mathrm{i}\}} & i=1,2, \ldots, N
\end{array}
$$

\section{Attraction and Repulsion}

To stay in the community, two "fish" which are far from each other may steer to come closer to each other (Attraction) and two "fish" which are "too close" to each other may repel each other to avoid collision (Repulsion). These basic behaviors are performed by dividing the area around each agent into four sections: Front, Rear, Left, and Right (see figure 3.4). In this case, each agent looks at its nearest neighbor in each section, and based on the spacing distance, decides what to do. If the nearest agent in the Front section is "far", the agent speeds up and if it is "too close", the agent slows down. Similar behaviour is performed for the other sections. Table 3.1 illustrates the different types of behaviour which may occur.

Figure 3.6: shows a representative behaviour of a group of 40 "fish" using the above type of decentralized control. In this case, figure 3.6 gives a sequence of time frames showing the step by step formation of a group of "fish" passing by a fixed obstacle. The leader is virtual and can pass through the obstacle. Frames are obtained by freezing the "movie" every two seconds. There are 39 "fish" each of them possessing nonlinear underwater vehicle dynamics. Each fish's behaviour is 
obtained by adding three actions. First, the fish find their heading angle by averaging the fish's heading within their neighborhood radius; second, they repel very close fish and attract farther (but not too far) fish in the four neighborhood sections (Right, Left, Front, Rear); third, they avoid an obstacle if they are inside the visibility region of the obstacle, and change their heading direction to the outgoing line from the center of the obstacle. They also "escape faster", if they are closer to the center of the obstacle. The obstacle can split the group into two slices depending on the initial conditions, the radius of neighborhood, and other parameters of the system. Although, there is no guarantee that the group of fish will remain as one "cluster", it can be seen in the above simulation that the group of "fish" split into two clusters about the obstacle, are thence re-group to form one cluster because of the attraction behaviour.

Table 3.1. Basic behavioural reactions of attraction and repulsion

\begin{tabular}{|l|l|l|l|l|}
\hline $\begin{array}{l}\text { Nearest } \\
\text { Neighbour }\end{array}$ & Left & Right & Front & Rear \\
\hline Too close & Steer to right & Steer to left & Slow down & Speed up \\
\hline Far & Steer to left & Steer to right & Speed up & Slow down \\
\hline out of range & No action & No action & No action & No action \\
\hline
\end{tabular}

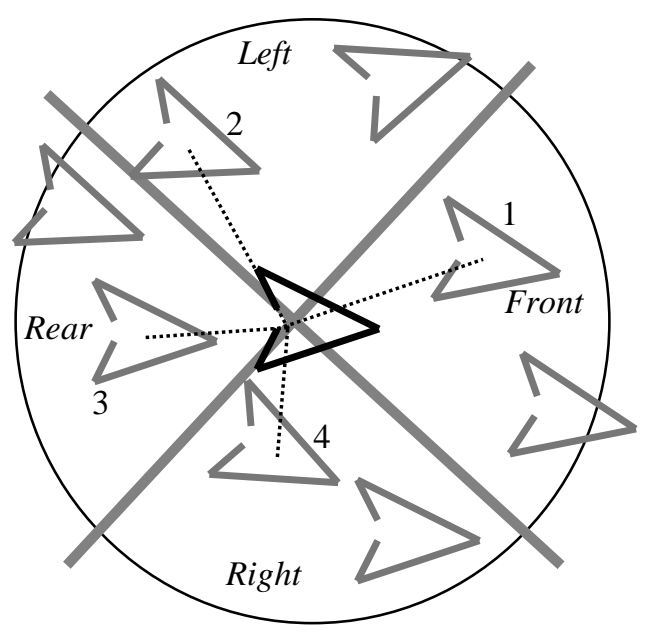

Fig 3.4. Sections and nearest neighbors in each section about a given agent. Here, agents 1, 2, 3, and 4 are the nearest neighbors occurring in the Front, Left, Rear, Right sections respectively. 


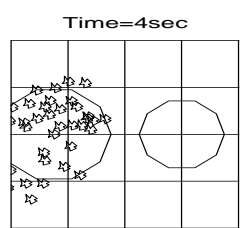

Time $=12 \mathrm{sec}$

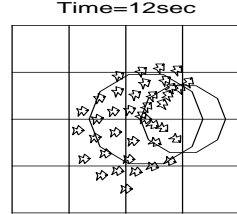

Time $=20$ sec

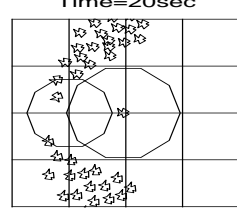

Time $=28 \mathrm{sec}$

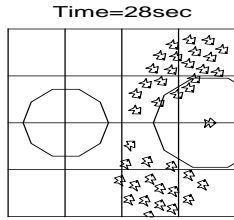

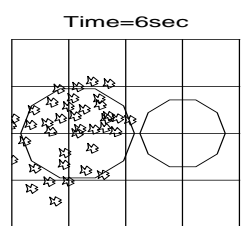

Time $=14 \mathrm{sec}$

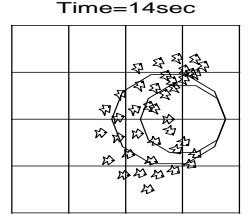

Time=22sec

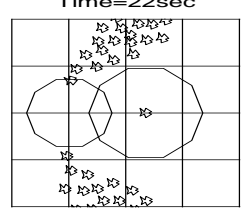

Time $=30$ sec

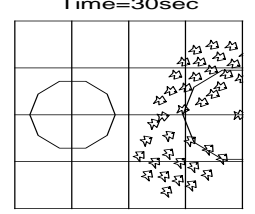

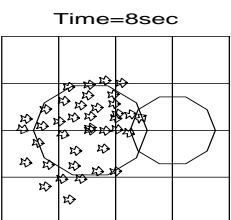

Time $=16 \mathrm{sec}$
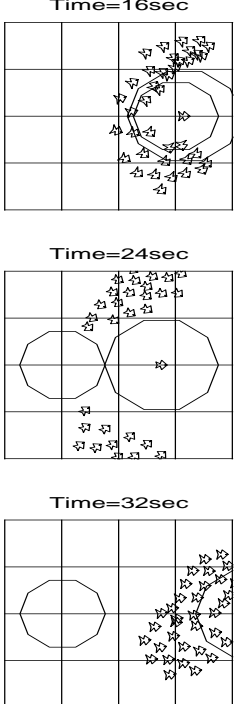

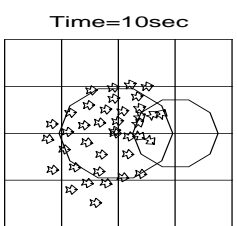

Time $=18 \mathrm{sec}$

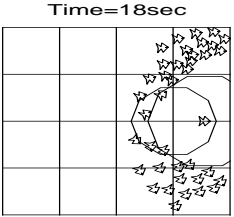

Time $=26 s e c$

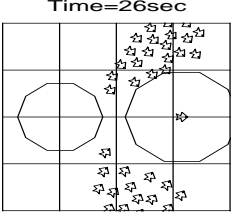

Time $=34 \mathrm{sec}$

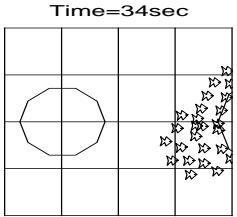

Fig 3.6. Step by step frames of the position and orientation of the group of fish while passing an obstacle.

\section{Example of Cooperative Control in Man-Made Systems}

We shall consider the decentralized control if a platoon of identical vehicles. Since there are various types of four-wheel vehicles which are a combination of the following columns:

\begin{tabular}{|l|l|}
\hline Drive System & Steering System \\
\hline Front Wheel Drive & Front Wheel Steering \\
\hline Rear Wheel Drive & Rear Wheel Steering \\
\hline Four Wheel Drive & Four Wheel Steering \\
\hline
\end{tabular}

It is necessary to assume some type of configuration for the vehicle. For the mathematical model presented here, it will be assumed that all four wheels are driven and steered. It will also be assumed that the wheel-base (the distance between front axis and rear axis) of the vehicle is longer than the vehicle's width, so that a simpler two-wheel drive and steer vehicle model (bicycle-like) will be 
accurate enough to represent the behaviour of the vehicle (see figure 4.1 for a description of the vehicle).

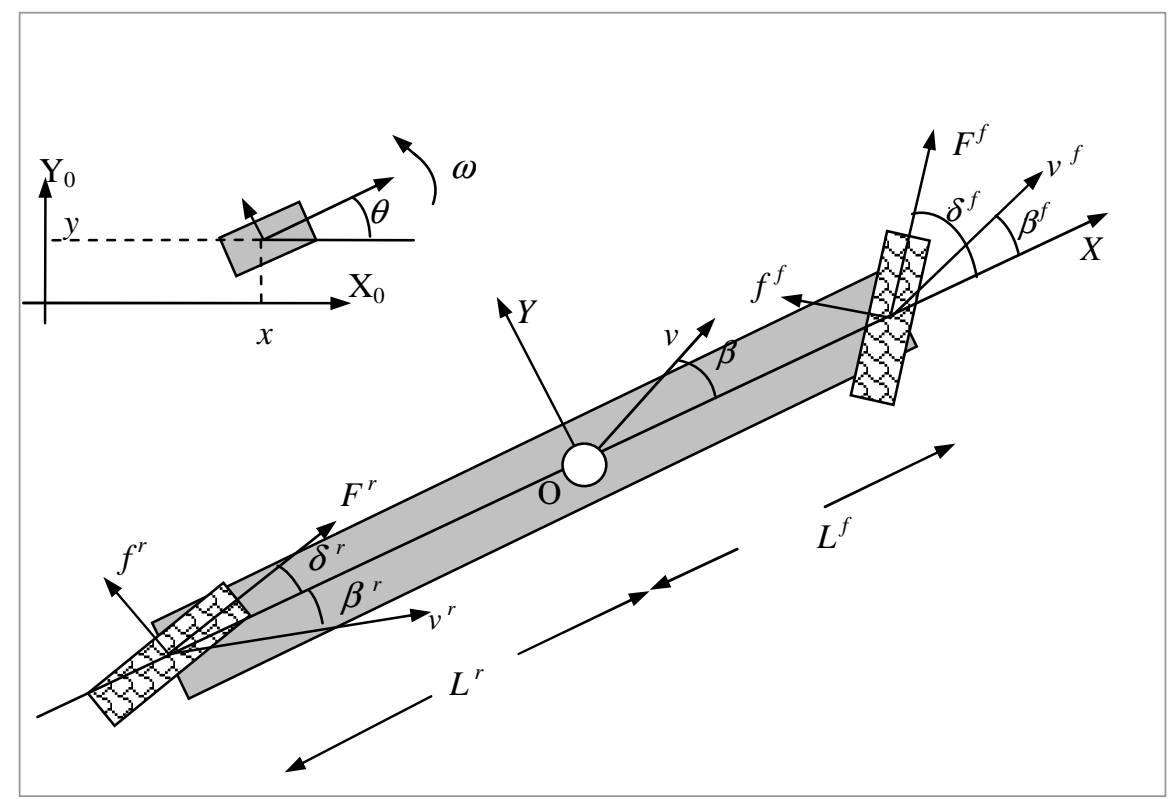

Fig 4.1. Single vehicle parameters and variables

In this figure, a description of the parameters is given in table 4.1. Figure 4.2 gives an illustration and description of the relevant parameters which exist between two adjacent vehicles, and in this figure, a description of the parameters is given in table 4.2).

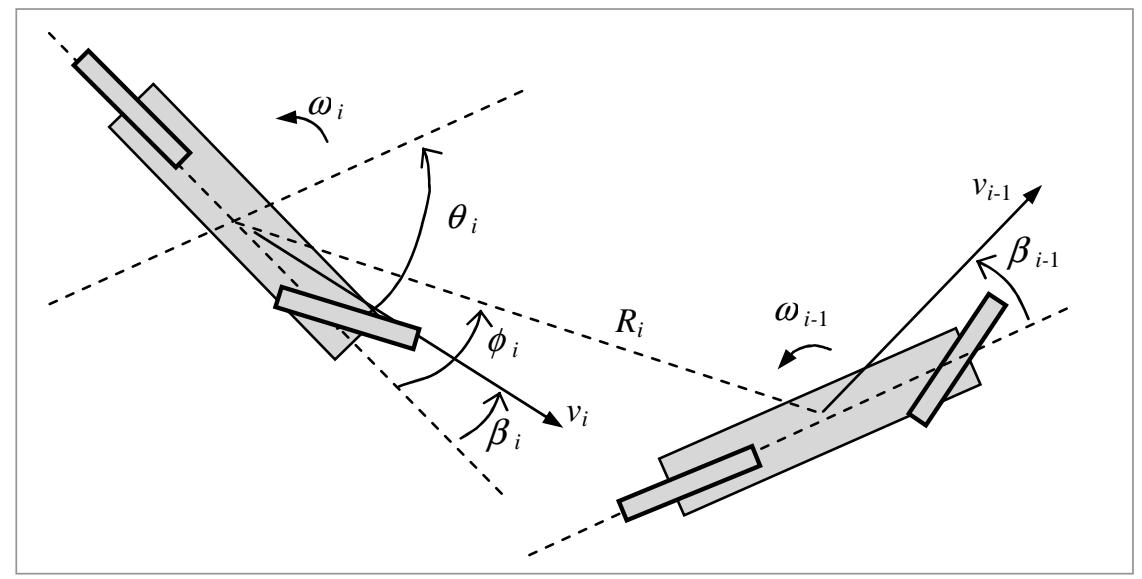

Fig 4.2. Relative variables between two adjacent vehicles. 
Table 4.1. Description of vehicle's parameters

\begin{tabular}{|l|l|}
\hline Parameter & Description \\
\hline$X$ & Absolute x-position \\
\hline$Y$ & Absolute y-position \\
\hline$\theta$ & Absolute heading angle \\
\hline$v$ & Longitudinal velocity of the center of mass \\
\hline$\omega$ & Angular velocity of the vehicle about its center of mass \\
\hline$\beta$ & Skidding angle \\
\hline$F^{f}$ & Front wheel traction force (Input) \\
\hline$F^{r}$ & Rear wheel traction force (Input) \\
\hline$\delta^{f}$ & Front wheel steering angle (Input) \\
\hline$\delta^{r}$ & Rear wheel steering angle (Input) \\
\hline$F^{f}$ & Front wheel lateral force \\
\hline$F^{r}$ & Rear wheel lateral force \\
\hline$\beta^{f}$ & Front wheel skidding angle \\
\hline$\beta^{r}$ & Rear wheel skidding angle \\
\hline$v^{f}$ & Front wheel longitudinal velocity \\
\hline$v^{r}$ & Rear wheel longitudinal velocity \\
\hline$L^{f}$ & Distance between Front wheel and the center of mass \\
\hline$L^{r}$ & Distance between Rear wheel and the center of mass \\
\hline
\end{tabular}

Table 4.2. Description of the parameters given in figure 4.2

\begin{tabular}{|l|l|}
\hline Parameter & Description \\
\hline$R_{i}$ & Relative Distance from the previous agent \\
\hline$\phi_{i}$ & $\begin{array}{l}\text { Angle between the heading direction and connector line } \\
\text { (connection angle) }\end{array}$ \\
\hline$\theta_{i}$ & $\begin{array}{l}\text { Relative heading angle (relative to the heading angle of } \\
\text { the previous agent) }\end{array}$ \\
\hline$v_{i}$ & Longitudinal velocity of the center of mass \\
\hline$\omega_{i}$ & Angular velocity of the vehicle about its center of mass \\
\hline$\beta_{i}$ & Skidding angle \\
\hline
\end{tabular}

Figure 4.3 shows the interaction which exists between the vehicles of a platoon.

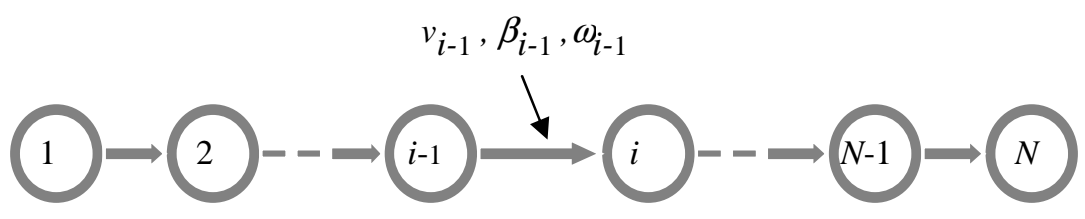

Fig 4.3. Interconnection between the vehicles of a platoon 
From the previous assumption made, the vehicle can be modeled as a bicycle-like vehicle. In the following models of the vehicle however, it is assumed that the vehicle is four-wheel-drive / four-wheel-steering, whereas in the numerical examples studied, it is assumed that the car is front-wheel-drive / front wheel steering.

\section{Nonlinear Model}

A nonlinear model of the four-wheel-drive / four wheel steering vehicle can be obtained as follows [10]:

$$
\left\{\begin{array}{l}
\dot{R}_{i}=-v_{i} \cos \left(\phi_{i}-\beta_{i}\right)+v_{i-1} \cos \left(\theta_{i}-\phi_{i}+\beta_{i-1}\right) \\
\dot{\phi}_{i}=\frac{1}{R_{i}} v_{i} \sin \left(\phi_{i}-\beta_{i}\right)-\omega_{i}+\frac{1}{R_{i}} v_{i-1} \sin \left(\theta_{i}-\phi_{i}+\beta_{i-1}\right) \\
\dot{\theta}_{i}=-\omega_{i}+\omega_{i-1} \\
m \dot{v}_{i}=f_{i}^{x} \cos \beta_{i}+f_{i}^{y} \sin \beta_{i} \\
\dot{\beta}_{i}=-\frac{1}{m v_{i}} f_{i}^{x} \sin \beta_{i}+\frac{1}{m v_{i}} f_{i}^{y} \cos \beta_{i}-\omega_{i} \\
\dot{\omega}_{i}=\frac{1}{J} \tau_{i}^{z}
\end{array}\right.
$$

where $B$ is the longitudinal damping ratio, $b$ is the rotational damping ratio, $m$ is the mass of the vehicle and $J$ is the moment of inertia,

where:

$$
\left\{\begin{array}{l}
f_{i}^{x}=-f_{i}^{f} \sin \delta_{i}^{f}+F_{i}^{f} \cos \delta_{i}^{f}-f_{i}^{r} \sin \delta_{i}^{r}+F_{i}^{r} \cos \delta_{i}^{r}-B v_{i} \cos \beta_{i} \\
f_{i}^{y}=f_{i}^{f} \cos \delta_{i}^{f}+F_{i}^{f} \sin \delta_{i}^{f}+f_{i}^{r} \cos \delta_{i}^{r}+F_{i}^{r} \sin \delta_{i}^{r}-B v_{i} \sin \beta_{i} \\
\tau_{i}^{z}=L^{f}\left(f_{i}^{f} \cos \delta_{i}^{f}+F_{i}^{f} \sin \delta_{i}^{f}\right)-L^{r}\left(f_{i}^{r} \cos \delta_{i}^{r}+F_{i}^{r} \sin \delta_{i}^{r}\right)-b \omega_{i}
\end{array}\right.
$$

where:

$$
\left\{\begin{array}{l}
f_{i}^{f}=f^{f}\left(\delta_{i}^{f}-\beta_{i}^{f}\right) \approx \mu^{f}\left(\delta_{i}^{f}-\beta_{i}^{f}\right) \\
f_{i}^{r}=f^{r}\left(\delta_{i}^{r}-\beta_{i}^{r}\right) \approx \mu^{r}\left(\delta_{i}^{r}-\beta_{i}^{r}\right)
\end{array}\right.
$$

and where $\mu^{f}$ and $\mu^{r}$ are linear approximation coefficients for the lateral friction forces $f^{f}$ and $f^{r}$. It is to be noted that the following approximation can be made for small $\beta$ :

$$
\left\{\begin{array}{l}
\tan \left(\beta_{i}^{f}\right)=\tan \left(\beta_{i}\right)+\frac{L^{f} \omega_{i}}{v_{i} \cos \beta_{i}} \Rightarrow \beta_{i}^{f} \approx \beta_{i}+\frac{L^{f} \omega_{i}}{v_{i}} \\
\tan \left(\beta_{i}^{r}\right)=\tan \left(\beta_{i}\right)+\frac{L^{r} \omega_{i}}{v_{i} \cos \beta_{i}} \Rightarrow \beta_{i}^{r} \approx \beta_{i}+\frac{L^{r} \omega_{i}}{v_{i}}
\end{array}\right.
$$




\section{Equilibrium Point}

The equilibrium point for the above set of nonlinear differential equations for each vehicle can be selected as:

$\left[\begin{array}{llllll}R_{e q} & \phi_{e q} & \theta_{e q} & v_{e q} & \beta_{e q} & \omega_{e q}\end{array}\right]^{\prime}=\left[\begin{array}{llllll}R^{0} & \phi^{0} & 0 & v^{0} & 0 & 0\end{array}\right]^{\prime}$

$\left[\begin{array}{llll}F_{e q}^{f} & \delta^{f} & F_{e q}^{r} & \delta_{e q}^{r}\end{array}\right]^{\prime}=\left[\begin{array}{llll}F^{f 0} & 0 & F^{r 0} & 0\end{array}\right]^{\prime}$

which is constrained to:

$F^{f 0}+F^{r 0}=B v^{0}$

and $v^{0}$ must be identical for all vehicles.

from which, the linearized model of the vehicle about this equilibrium point is obtained as (4.4).

In the simulations to be carried out, the controller used to control the distance between a vehicle and its immediate neighbouring vehicle is assumed to be a three-term controller $(P I D)$, which can provide a zero steady state tracking error. To have string stability, the controllers must be non-identical [2].

The controller used to control the connection-angle $(\phi)$ is assumed to be a simple P-controller which provides approximate tracking for the reference signal $\left(\phi^{0}\right)$. In the following simulations there are 19 cars following the lead vehicle where the lead vehicle follows a specified reference path. In this case, the results of figure 4.5 are obtained, where the input profile applied to the lead vehicle is given in figure 4.4 .

$$
\begin{aligned}
& {\left[\begin{array}{c}
\Delta \dot{R}_{i} \\
\Delta \dot{\phi}_{i} \\
\dot{\theta}_{i} \\
\Delta \dot{v}_{i} \\
\Delta \dot{\beta}_{i} \\
\Delta \dot{\omega}_{i}
\end{array}\right]=\left[\begin{array}{cccccc}
0 & 0 & v_{i}^{0} \sin \varphi_{i}^{0} & -\cos \phi_{i}^{0} & -v_{i}^{0} \sin \varphi_{i}^{0} & 0 \\
0 & 0 & \frac{v_{i}^{0}}{R_{i}^{0}} \cos \phi_{i}^{0} & \frac{1}{R_{i}^{0}} \sin \phi_{i}^{0} & -\frac{v_{i}^{0}}{R_{i}^{0}} \cos \phi_{i}^{0} & -1 \\
0 & 0 & 0 & 0 & 0 & -1 \\
0 & 0 & 0 & -B / m & 0 & 0 \\
0 & 0 & 0 & 0 & -\frac{1}{m v_{i}^{0}}\left[\mu^{f}+\mu^{r}+B v_{i}^{0}\right] & -1+\frac{1}{m v_{i}^{02}}\left[-\mu^{f} L^{f}+\mu^{r} L^{r}\right] \\
0 & 0 & 0 & 0 & \frac{1}{J}\left[-\mu^{f} L^{f}+\mu^{r} L^{r}\right] & -\frac{1}{J v_{i}^{0}}\left[\mu^{f} L^{f}+\mu^{r} L^{r 2}\right]-\frac{b}{J}
\end{array}\right]\left[\begin{array}{c}
\Delta R_{i} \\
\Delta \phi_{i} \\
\Delta \theta_{i} \\
\Delta v_{i} \\
\Delta \beta_{i} \\
\Delta \omega_{i}
\end{array}\right]} \\
& +\left[\begin{array}{cccc}
0 & 0 & 0 & 0 \\
0 & 0 & 0 & 0 \\
0 & 0 & 0 & 0 \\
\frac{1}{m} & 0 & \frac{1}{m} & 0 \\
0 & \frac{1}{m v_{i}^{0}}\left[\mu^{f}+F_{i}^{f 0}\right] & 0 & \frac{1}{m v_{i}^{0}}\left[\mu^{r}+F_{i}^{r 0}\right] \\
0 & \frac{L^{f}}{J}\left[\mu^{f}+F_{i}^{f 0}\right] & 0 & -\frac{L^{r}}{J}\left[\mu^{r}+F_{i}^{r 0}\right]
\end{array}\right]\left[\begin{array}{c}
\Delta F_{i}^{f} \\
\Delta \delta_{i}^{f} \\
\Delta F_{i}^{r} \\
\Delta \delta_{i}^{r}
\end{array}\right]+\left[\begin{array}{ccc}
\cos \phi_{i}^{0} & v_{i}^{0} \sin \varphi_{i}^{0} & 0 \\
-\frac{1}{R_{i}^{0}} \sin \phi_{i}^{0} & \frac{v_{i}^{0}}{R_{i}^{0} \cos \phi_{i}^{0}} & 0 \\
0 & 0 & 1 \\
0 & 0 & 0 \\
0 & 0 & 0 \\
0 & 0 & 0
\end{array}\right]\left[\begin{array}{c}
\Delta v_{i-1} \\
\Delta \beta_{i-1} \\
\Delta \omega_{i-1}
\end{array}\right]
\end{aligned}
$$


Figure 4.6 shows the response of the string of the vehicles with respect to the same maneuver of the leader as given in figure 4.4. For identical controllers, the relative distance is string unstable (case (a) in figure 4.6) and for non-identical controllers the response is string stable (case (b) in figure 4.6).

Steering angle $\delta^{f}$

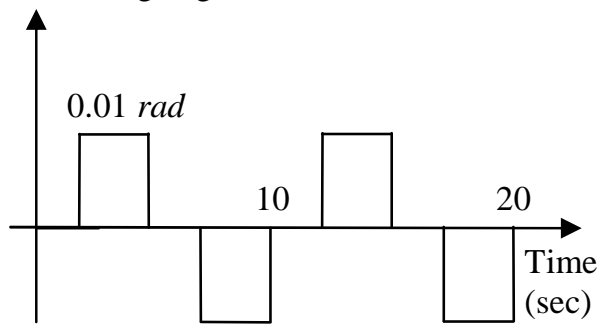

(a)
Traction Force $F^{f}$

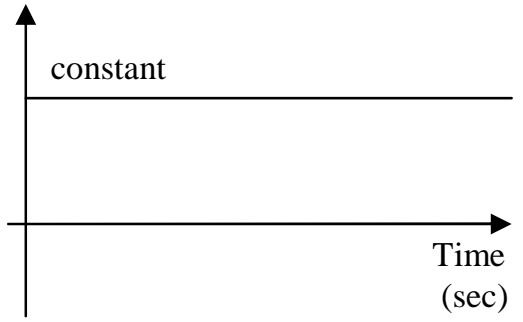

(b)

Fig 4.4. The input profile applied to the leader:

Time $=$ Osec

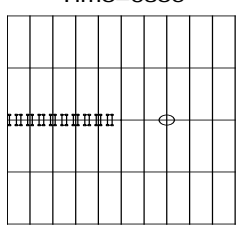

Time $=12 \mathrm{sec}$

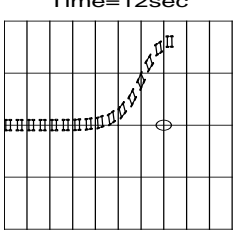

Time $=24 \mathrm{sec}$

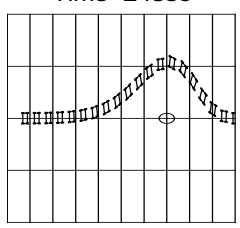

Time $=36 \mathrm{sec}$

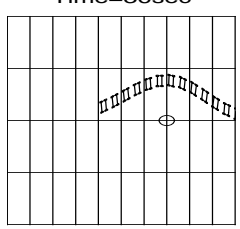

Time $=3 \mathrm{sec}$

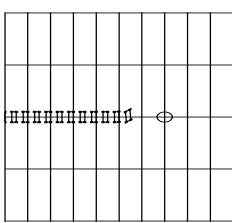

Time $=15 \mathrm{sec}$

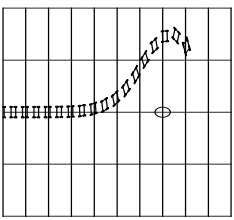

Time $=27 \mathrm{sec}$

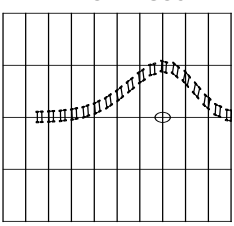

Time $=39 \mathrm{sec}$

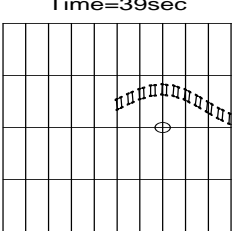

Time $=6 \mathrm{sec}$

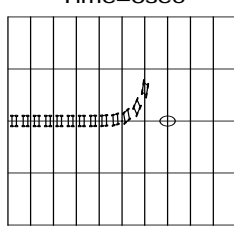

Time $=18 \mathrm{sec}$

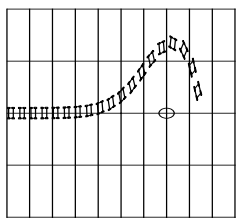

Time $=30$ sec

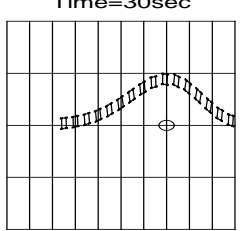

Time $=42 \mathrm{sec}$

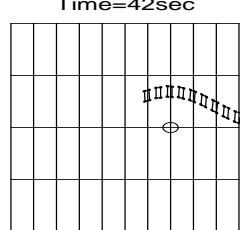

Fig 4.5. Cars passing an obstacle.

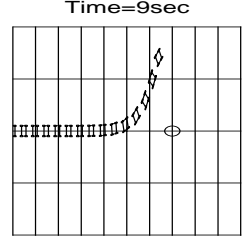

Time $=21 \mathrm{sec}$

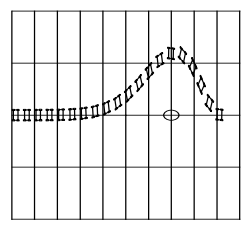

Time $=33 \mathrm{sec}$
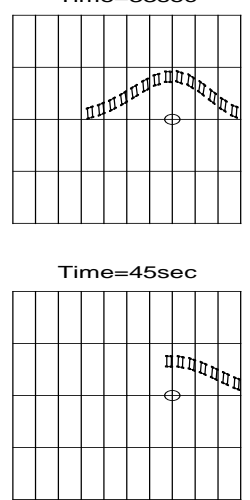


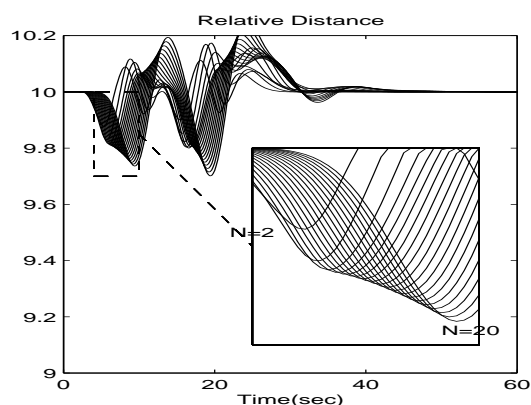

(a)

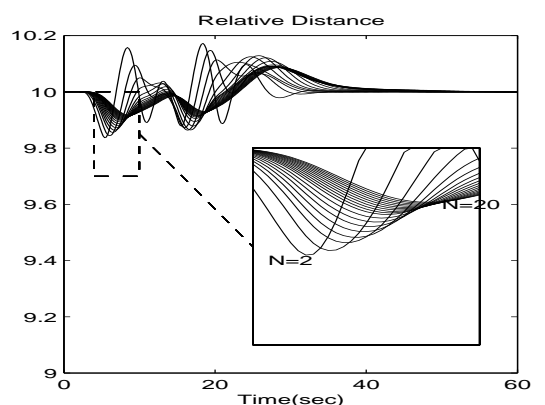

(b)

Fig 4.6. String unstable response for case of identical controllers (a) v.s. String stable response (b) for case of non-identical controllers

\section{Example of cooperative control in the human body}

\subsection{Intestinal Slow-Wave}

In the intestinal tract of mammals, it has been observed that there exists an intestinal slow-wave frequency gradient observed in situ, where the frequency decreases aborally in step-wise fashion, with each frequency plateau being separated from the next by an area of waxing and waning, e.g. see [3], [4] Here figures 5.1, 5.2, and 5.3 show the type of behaviour, which has been observed.

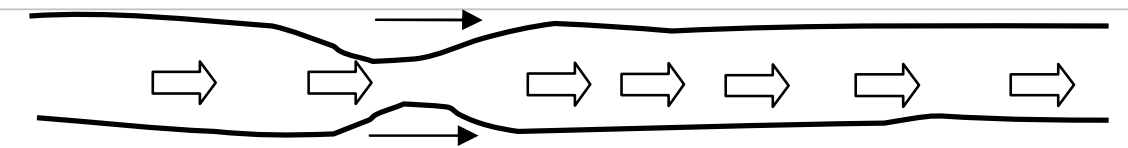

Fig 5.1. Intestinal Slow-Wave

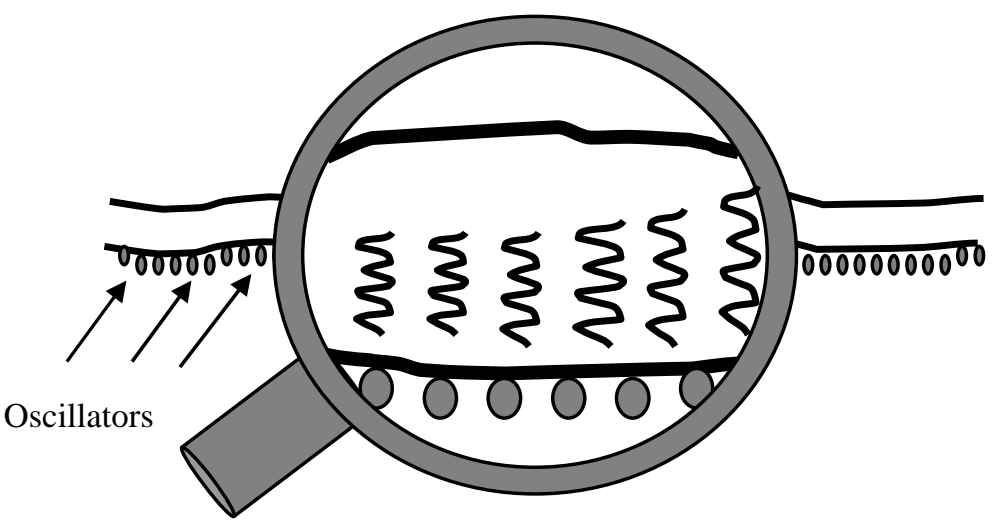

Fig 5.2. Intestinal oscillators 


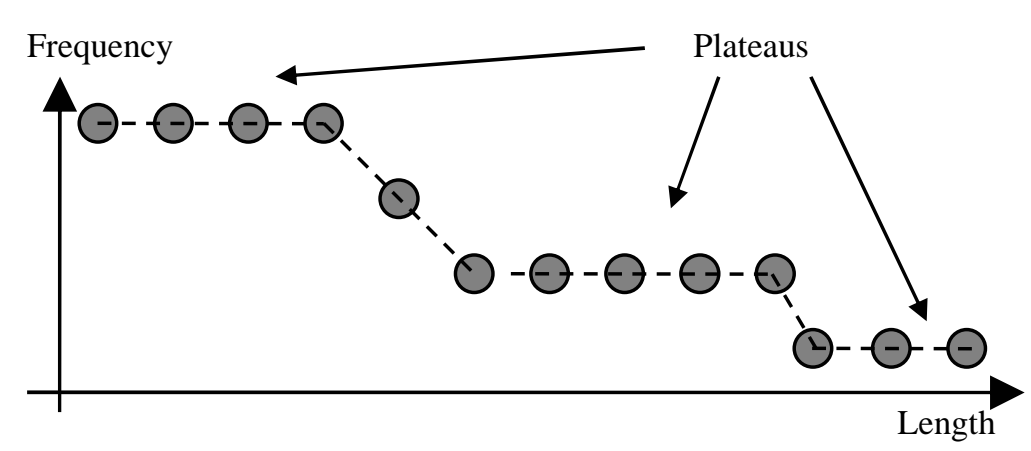

Fig 5.3. Plateaus of frequencies of oscillators with respect to the length of the intestine.

In this case, it has been pointed out, e.g. see [11], that the intestinal slow wave closely resembles a "relaxation oscillator", in which a series of such near-identical oscillators are interconnected together, as illustrated in figure 5.4.

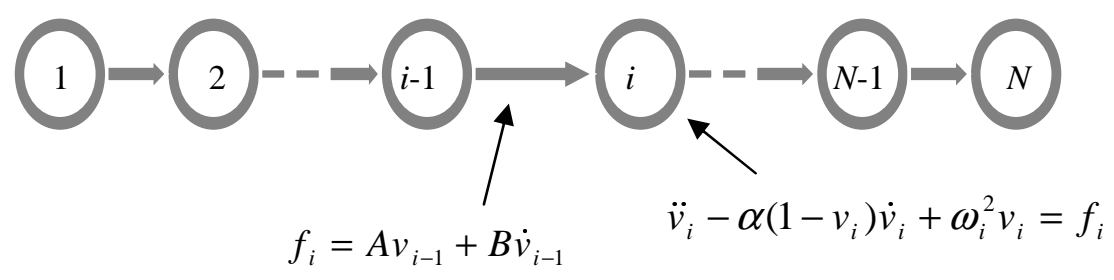

Fig 5.4. Van Der Pol interconnected oscillators

In [3], it was shown that a series of Van Der Pol relaxation oscillators of successively decreasing frequencies, with "small" coupling terms interconnecting the oscillators, as illustrated in figure 5.5, could produce the experimentally observed plateau effect.

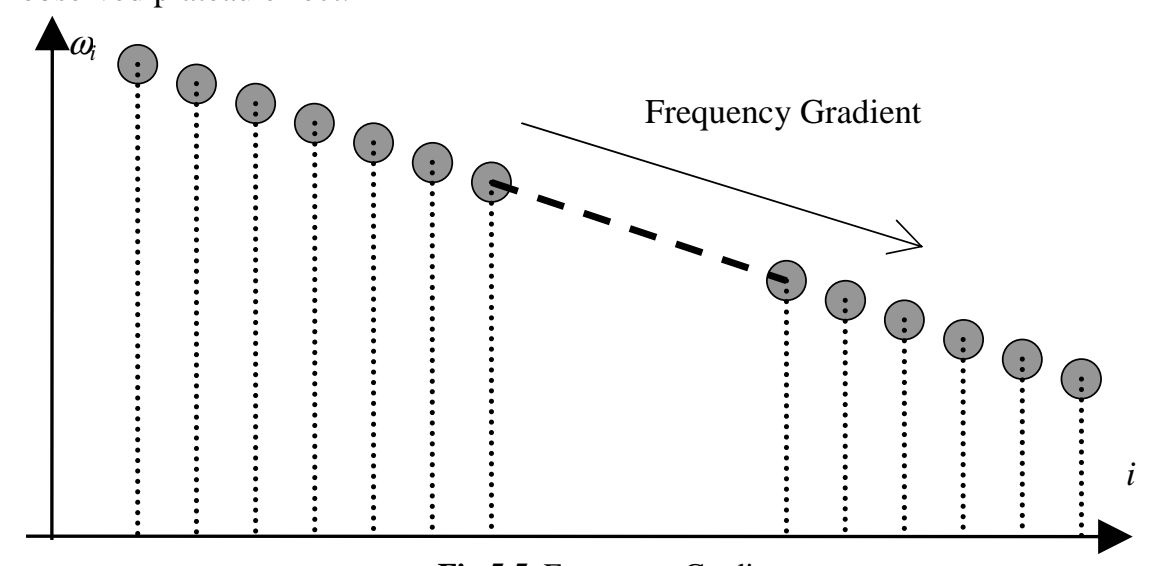

Fig 5.5. Frequency Gradient 
For example, if 10 oscillators of the type:

$\ddot{v}_{i}-\alpha\left(1-v_{i}\right) \dot{v}_{i}+\omega_{i}^{2} v_{i}=f_{i} \quad, i=1,2, \ldots, 10$

where:

$$
f_{i}=A v_{i-1}+B \dot{v}_{i-1} \quad, i=2,3, \ldots, 10
$$

with $\alpha=2.5 ; A=0 ; B=0.5$;

$\omega=\left(\omega_{1}, \omega_{2}, \cdots, \omega_{10}\right)^{\prime}=(20.5,16.5,16,15.5,15,14.5,14,13.5,13,12.5)^{\prime}$; then in steady-state, the resultant frequencies of the corresponding oscillators are given by figure 5.6. In his case, the same qualitative behaviour, as observed experimentally with respect to various frequency plateaus, are obtained, and the resultant frequencies decrease in a step-wise fashion, as also has been observed experimentally.

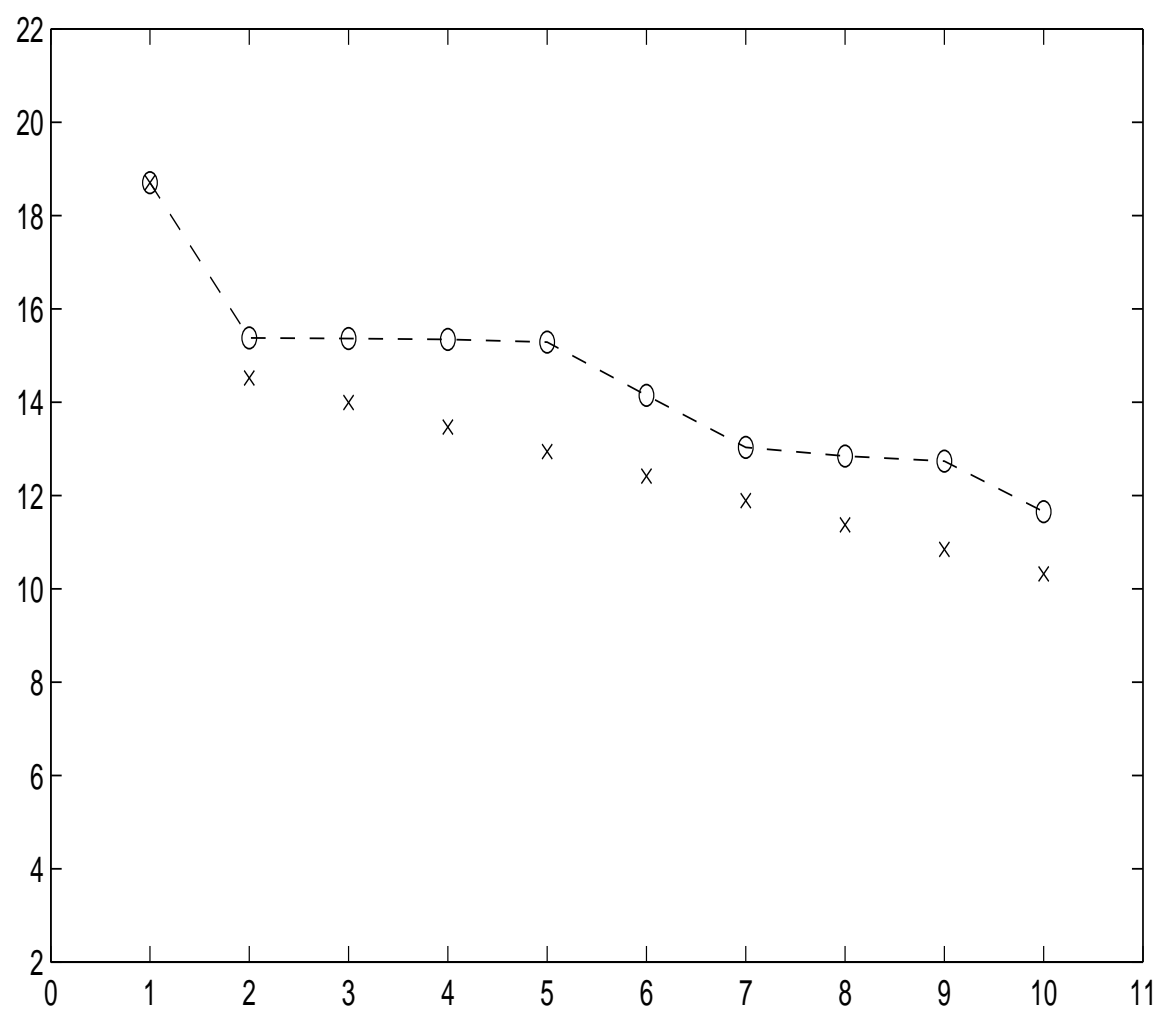

Fig 5.6. Frequency plateaus obtained; uncoupled oscillators are marked as (x) and coupled oscillators are marked as (o)

Moreover in the case of the coupled oscillators, the same type of waxing and waning which has been observed experimentally, is now obtained in the simulations. For example, figure 5.6 gives some representative time simulations 
for the system (5.1)-(5.2) for the case of (a) uncoupled oscillators and (b) coupled oscillators, and "waxing and waning" is observed in (b).

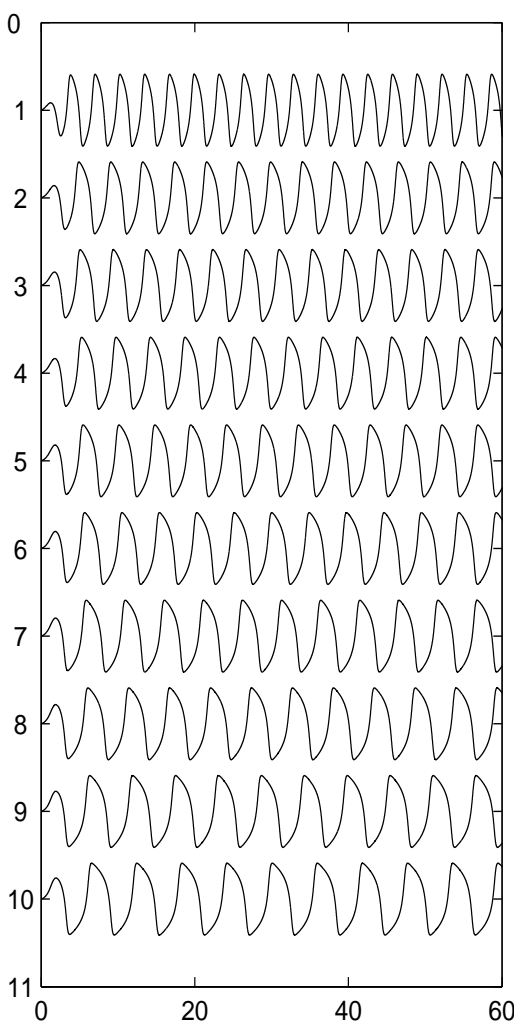

(a)

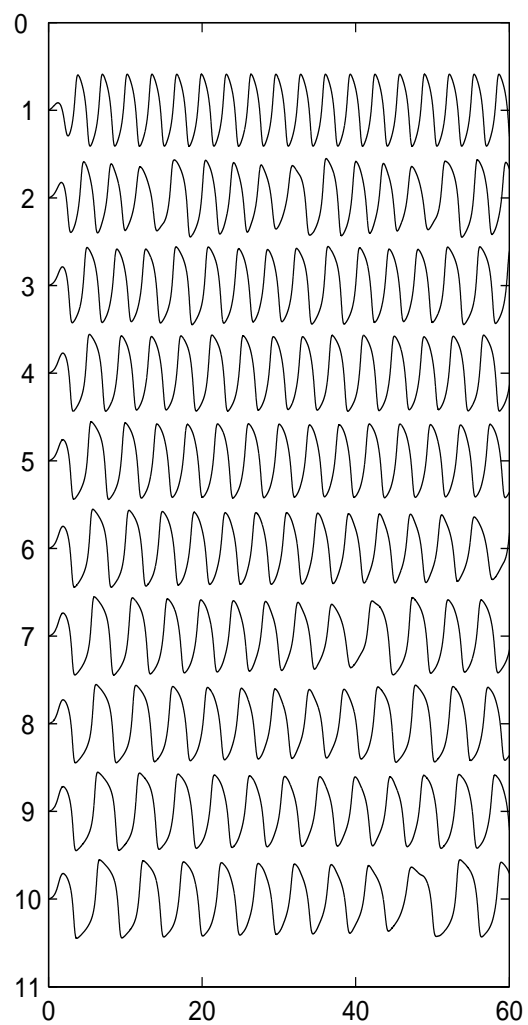

(b)

Fig 5.6. Uncoupled (a) and coupled (b) Van Der Pol oscillator outputs. Note that waxing and waning can be observed in (b).

\section{Conclusions}

This paper has given an overview of some of the properties and some of the problems which arise when the decentralized control of large numbers of identical or near-identical objects are interconnected together in various configurations. In particular, three classes of problems are studied: the behaviour of a group of fish, of a group of vehicles, and the behaviour of the intestinal slow-wave signal propagation in a human body. There are many questions which arise in such application problems, and much study is still required to resolve these questions. 


\section{References:}

1. D. Swaroop, J.K. Hedrick, "String Stability of Interconnected Systems", IEEE Transactions on Automatic Control, vol 41, no 3, 1996, pp 349-357.

2. M. E. Khatir, E. J. Davison, "Decentralized Control of a Large Platoon of Vehicles Using Non-Identical Controllers", American Control Conference 2004 (Submitted)

3. N. E. Diamant, P.K. Rose, E. J. Davison, "Computer Simulation of Intestinal slowwave frequency gradient", American Journal of Physiology, vol.219, no. 6, Dec. 1970, pp.1684-1690.

4. N. E. Diamant, A. Bortoff, "Nature of the Intestinal slow-wave frequency gradient", American Journal of Physiology, vol.219, 1969, pp.301-307.

5. F. V. Palmay, E. J. Davison, J. Duffin, "The Simulation of Multi-Neurone Networks: Modelling of the Lateral Inhibition of the Eye and the Generation of Respiratory Rhythm”, Bulletin of Mathematical Biology, Volume 36, 1974, pp. 77-99.

6. A. Jadbabaie, J. Lin, A. S. Morse, "Coordination of Groups of Mobile Autonomous Agents Using Nearest neighbor Rules", IEEE Transactions on Automatic Control, Vol. 48, No. 6, June 2003, pp. 988-1001.

7. Y. Liu, K. M. Passino, M. M. Polycarpou, "Stability analysis of M-Dimensional Asynchronous Swarms With a Fixed Communication Topology", IEEE Transactions on Automatic Control, Vol. 48, No.1, January 2003, pp. 76-95.

8. V. Gazi, K. M. Passino, "Stability Analysis of Swarms", IEEE Transactions on Automatic Control, Vol. 48, No. 4, April 2003, pp. 692-697.

9. A. Das, R. Fierro, V. Kumar, J. Ostrowski, J. Spletzer, C. Taylor, “ A Framework for Vision Based Formation Control", IEEE Transactions on Robotics and Automation, Vol. 18, No. 5, Oct. 2002 pp. 813-825 .

10. Jurgen Ackermann, "Robust Control: Systems with Uncertain Physical Parameters", Springer-Verlag, London Limited, 1993.

11. M. E. Khatir, E. J. Davison, "Cooperative Control of Large Systems", Dept. of Electrical and Computer Engineering, University of Toronto, Systems Control Group Report No. 0301, March 2003. 